\title{
Evaluation of antibiotic consumption in Clinical hospital in Stip for the period 2017-2019
}

\author{
Biljana Lazarova $^{1}$, Biljana Eftimova ${ }^{1}$, Lidija Mihailova $^{1}$, Sanja Filkova ${ }^{2}$, \\ Zorica Naumovska ${ }^{3}$, Zoran Sterjev ${ }^{3}$ \\ ${ }^{1}$ Clinical hospital, Ljuben Ivanov bb, 2000 Stip, North Macedonia \\ ${ }^{2}$ Department of Clinical Pharmacy, Mother Theresa 17, 1000 Skopje, North Macedonia \\ ${ }^{3}$ Faculty of Pharmacy, Ss. Cyril and Methodius University, Mother Theresa 47, 1000 Skopje, North Macedonia
}

\section{Introduction}

Antibiotics are widely used in clinical practice globally and their irrational use could lead to antimicrobial resistance and negative therapeutic outcome even in a case of easy to treat diseases. The misuse and overuse use of antibiotics additionally initiate negative effects on the normal bacterial flora in patients, triggering superinfections, adverse event occurrence and subsequently increase of treatment costs. One of the most important fact for irrational antibiotic utilization is the negative impact on the hospital microbial environment erasing the inevitable need for permanent monitoring of the spread of bacterial resistance in everyday clinical practice (Cizman et al., 2003). Nowadays healthcare professionals face serious problem while infections caused by resistant microorganisms gradually increase, antimicrobial options diminish and global market lacks new antibiotics. Monitoring the antibiotic consumption and prescription pattern are critical due to the fact that they could provide valuable data for adherence to globally accepted guidelines in clinical practice.

The aim of this study is to evaluate antibiotic consumption in the Clinical hospital in Stip, with the
ATC/DDD index, which is an accepted standard method in three years period 2017-2019.

\section{Materials and methods}

A retrospective study was carried out at the Clinical hospital in Stip, in order to evaluate the antibiotic consumption in period 2017-2019. The data were obtained for the following ten departments (internal medicine, neurology, infectious diseases, general surgery, orthopedics, urology, gynecology, obstetrics, pediatrics, intensive care unit) with total of 288 beds. Antibiotics utilization was evaluated based on dispensed antibiotics with ATC code J01 (regardless of the indication for which they were used - prophylaxis or therapy) for each department provided from the hospital pharmacy. The ATC/DDD index is a universal parameter used in the evaluation of antibiotic use (Filius et al., 2005).

Total number of hospital days (bed/day) BD, during a certain period of time and the index of occupancy of hospital beds were collected from statistics department in the Clinic. The DDD is the assumed average maintenance dose per day for a drug used for its main indication in adults. The quantity of antibiotics was converted into a number

\footnotetext{
*blazarova@yahoo.com
} 
of defined daily doses (DDD/100 bed-days (DBD) via the anatomical-therapeutic-chemical (ATC) and DDD drug classification. DDD values of every antibiotic is calculated separately with Antibiotic consumption calculator (ABC Cal Version 3.1constructed by Monnet DL, Staten Serum Institute 2006. DDDs = Number of boxes $\mathrm{x}$ number of tablets in the box or number of vials $\mathrm{x}$ grams of active compound in tablet or vial/the DDD value of the antibiotic in grams.In this calculation method, the form used for in-bed patients is the ratio of the total DDD per 100-bed-days. This index is called antimicrobial consumption index (ACI). ACI=DDDs/bed-days $\times 100$ (Kuster et al., 2008; WHO, 2010).

\section{Results and discussion}

In our study, cephalosporins were found to be the most frequently used antimicrobials in all evaluated departments with the highest consumption of 3rd generation cephalosporin, ceftriaxone. The highest utilization of ceftriaxone with over $94.5 \%$ and $i=0.50$ was confirmed in the departments for gynecology. On the internal medicine department, ceftriaxone accounts $82 \%$ with $\mathrm{i}=0.89$ in 2017; $71.4 \%$; $i=0.79$ in 2018 and $41.59 \%$ with $i=0.59$ in 2019. This declination observed for ceftriaxone, resulted in increased consumption of ciprofloxacin with $37.2 \%$ compared to year $2018(14.05 \%)$ and $2017(12.6 \%)$. The same pattern in evaluated three year period was confirmed, in the neurology, pediatrics, infective and urology departments, with increased utilization of fluoroquinolones. On the neurology department, ceftriaxone accounts for $94.4 \%$ with $\mathrm{i}=0.79$ in $2017,94.7 \%$; $\mathrm{i}=0.62$ in 2018 and $85.99 \%$ with $i=0.62$ in 2019 . The increase of fluoroquinolone ciprofloxacin was also confirmed on this department with account of $2.43 \%, 3.82 \%$ and $7.54 \%$ for 2017,2018 and 2019 respectively. Increase from $8.07 \%$ to $12.94 \%$ was observed in the department for infectious diseases for ciprofloxacin and decrease from $83.78 \%$ to $76.71 \%$ with $i=0.52$ for cephalosporin from 2017 to 2019. Metronidazole was the second most frequently utilized antiinfective with $10.63 \%$ to $12.22 \%$ and $i=0.04$ for the evaluated period in the intensive care unit. Significant decrease of clindamycin consumption was confirmed in the general surgery department in evaluated period and increase of metronidazole use in $2017,5.99 \%$ and $\mathrm{i}=0.39$ compared to 2019 with $9.64 \%$ and $i=0.54$. Same pattern was confirmed in orthopedic department in three year period. The frequent use of cephalosporins and fluoroquinolones lead to the emergence of resistant microorganisms, thus problems such as the emergence of resistant pathogenes in our area would be an inevitable consequence. As antibiotics are the most frequently used drugs in the hospitals they constitute an important part of the total drug expenditure.

\section{Conclusion}

Utilization of ATC/DDD system in hospitals can provide internationally valid data in the evaluation of antimicrobial use. These data should result in more efficient antibiotics utilization in hospitals, and decisions made in line with the recommendations in established guidelines. Reducing irrational antibiotics use would prevent the incidence of lifethreatening serious infections, also preventing long hospital stays and higher health expenditures.

\section{References}

Cizman, M., 2003. The use and resistance to antibiotics in the community. Int. J. Antimicrob. Agents 21, 297307.

Filius, P.M.G., Liem, T.B.Y., van der Linden, P.D., Janknegt, R., Natsch, S., Vulto, A.G., Verbrugh, H.A., 2005. An additional measure for quantifying antibiotic use in hospitals. J. Antimicrob. Chemother. 55(5), 805-808.

Kuster, S.P., Ruef, C., Ledergerber, B., Hintermann, A., Deplazes, C., Neuber, L., 2008. Quantitative antibiotic use in hospitals: comparison of measurements, literature review and recommendations for a standard of reporting. Infection 36, 549-559.

World Health Organization (WHO), 2010. Collaborating Center for Drug Statistics Methodology: Guidelines for ATC classification and DDD assignment 2010. Available at: http://www.whocc.no/atcddd/.

Maced. Pharm. Bull. 66 (Suppl 1) 77 - 78 (2020) 